\title{
INTELIGENCIA: Del Campo Militar al Campo DE LOS Negocios
}

\author{
Mg. Nicko Gomero Gonzales \\ Director de la Oficina de Administración de la Facultad de Ciencias Contables
}

\begin{abstract}
RESUMEN
De un tiempo a esta parte hemos podido apreciar el cambio brusco que han sufrido nuestras sociedades contemporáneas. Por ello hay la necesidad de vivir a tono con los tiempos modernos que nos toca vivir, ya no basta la aplicación de modernos paradigmas, sino que hay la necesidad de un componente adicional que complemente el esfuerzo de las organizaciones, se trata del concepto de inteligencia empresarial.

La única manera de obtener una ventaja competitiva es mediante la innovación y la actualización. La inteligencia en una empresa es una base de datos, la inteligencia es información reunida para la elaboración de políticas de gobierno que iluminarán el campo de las opciones disponibles para la toma de decisiones.
\end{abstract}

Sin duda, la inteligencia aplicada a los negocios es una de las columnas del mercado actual.

Palabras clave: Innovación, cambio, tecnología, manejo estratégico, actualización, competitividad, inreligencia en los negocios.

\section{INTRODUCCIÓN}

En tiempos de rápidos cambios y avances prodigiosos, la competencia es cada vez más difícil e implacable. Es aquí donde un manejo apropiado y oportuno de la información cumple un papel de vital importancia como apoyo de las organizaciones. Ante las nuevas realidades de competitividad empresarial, no basta ya con la aplicación de los modernos paradigmas como el kaizen o mejoramiento continuo, el justo a tiempo, la calidad total, la planeación estratégica, el benchmarking; en fin, con toda la gama de esfuerzos internos por controlar los procesos, reducir los costos, mejorar la productividad, el producto y la gestión.

Cuando las necesidades de los clientes cambian rápidamente, cuando los produc- tos quedan obsoletos o no satisfacen totalmente los nuevos requerimientos de los consumidores, cuando las innovaciones tecnológicas se incorporan al mercado, cuando la competencia crece prácticamente sin control y sin fronteras, cuando existen definidos adversarios apoyados en el espionaje industrial, entonces, se requiere de un componente adicional que complemente el esfuerzo de las organizaciones, ya que las leyes ordinarias del mercado no permiten por sí solas explicar el éxito o el fracaso. Se trata del concepto de la inteligencia empresarial, también conocida como inteligencia competitiva o business intelligence.

La única manera de tener una ventaja competitiva es mediante la innovación y la actualización, la cual tiene en el manejo estratégi- 
co de la información un soporte fundamental, porque no sólo es poseer el conocimiento lo que permite determinar una ventaja, sino el tener la capacidad de hacer que dicho conocimiento sea utilizado de la forma más extensa y eficiente en todo el sistema corporativo a través de una integración interfuncional. De ahí que la forma de hacer algo conduzca a conseguir algo mejor. Se hace énfasis en lo referente a la información útil que es la que requieren los diferentes niveles de decisión de las organizaciones, que a su vez son los que producen resultados, con el fin de desarrollar coherentemente las estrategias y tácticas que logren los objetivos, mejorando simultáneamente la posición competitiva.

Erróneamente se pensaba que la inteligencia sólo era útil para asuntos de defensa y seguridad nacional, para prevenir amenazas o desarticular conflictos que ponían en peligro la integridad de la nación, pero no es así, como ya se vio en las primeras líneas de esta fase introductoria, también es aplicada en el mundo de los negocios, que es justamente lo que pretende demostrar el presente artículo, al desarrollar el marco teórico y su aplicación en este mundo que cada día se hace más competitivo.

\section{DEFINICIÓN DE INTELIGENCIA}

La palabra inteligencia proviene del latín Intelligeri, es decir, Inter-legere o Interligar. Por medio de su inteligencia el hombre llega a conocer la verdadera naturaleza del mundo que lo rodea, a sí mismo y todas las cosas que perciben sus sentidos; compara estas cosas, las clasifica, las distingue y las relaciona unas a otras formando así conceptos e ideas, siempre abstractos y generales para poder accionar sobre lo particular y lo concreto.
Para los antiguos griegos las más altas virtudes del hombre consistían en ser «bueno y hermoson; para los antiguos persas, en ser "veraz y valeroso"; para los teutones, en ser «fiel", y para nuestros contemporáneos, en ser «inteligente». Con el complejo significado de que este término ha sido reconocido desde hace miles de años.

La palabra inteligencia se deriva de la palabra latina intelligeri, que significa literalmente "recolectar de entre" y abarca los conceptos de percepción, discernimiento, selección y establecimiento de relaciones. En lengua hebrea hay muchas palabras distintas para designar el concepto de inteligencia, cada una de ellas con otras connotaciones: una resalta, igual que en latín, las características de discernimiento, de distinción; otra está relacionada con el concepto de máquina y de invento; una de estas palabras hebreas subraya el concepto de espacio, de amplitud y tolerancia, mientras otra recalca las características de la percepción en general y del gusto en particular; una palabra tiene el significado positivo de éxito y prosperidad, otra el negativo de complot $\mathrm{o}$ ardid.

Todavía otra palabra hebrea da a la inteligencia una connotación social de ayuda, consejo y progreso, mientras otra acentúa la firmeza y el poder. Todos estos matices y connotaciones del concepto de inteligencia hacen casi imposible definir el término. La idea moderna de inteligencia varía, necesariamente, de la antigua, aunque el valor atribuido a cada concepto depende de la concepción del mundo y de los requerimientos sociales de cada época.

Es característico el hecho de que en todas las connotaciones arriba mencionadas faltan algunas que son predominantes en nuestra época, como la memoria, el aprendizaje y el conocimiento. Los antiguos, por el contrario, describían el peligro psicológico que en- 
cerraban las relaciones entre la inteligencia y el conocimiento, como lo demuestra el vívido relato bíblico de la creación del mundo. La serpiente, representando al animal más inteligente, seduce al hombre para que adquiera el conocimiento comiendo el fruto prohibido, en el que están combinados el bien y el mal. El discernimiento mediante el conocimiento fue considerado como la causa de que el hombre perdiese su unión con el universo.

El valor positivo de la inteligencia tal como aparece en las más antiguas escrituras, fue la capacidad de establecer relaciones, su aplicación a una labor productiva social o inventiva, su integración en otras manifestaciones del organismo, tales como las sensaciones y la fuerza.

La inteligencia no es lo mismo que la pericia, pues un acto inteligente puede tener lugar sin prácrica y, al contrario, a pesar de la práctica, pueden realizarse actos poco inteligentes. Por último, la inteligencia no es una facultad aislada ni una respuesta estática a un estímulo.

En sí, el significado de inteligencia es muy complejo. Está estrechamente relacionado con el intelecto, término que abarca los conceptos de observación, pensamiento, capacidad de establecer relaciones, recuerdo e integración. La inteligencia es un estado de «agudeza mental" que nos permite resolver un problema y conseguir un fin; es una "capacidad para actuar y no un mecanismo para establecer relaciones". El acto inteligente depende del fin a que va dirigido.

\section{INTELIGENCIA EMPRESARIAL}

La inteligencia en una empresa no es una bases de datos. La inteligencia es información reunida para los elaboradores de políticas de gobierno, que ilumina el campo de las opciones disponibles para la toma de decisiones.
En su descripción más básica, la inteligencia en los negocios es la captación, selección, análisis y distribución de información convertida en inteligencia o conocimiento explícito para cambios favorables de la empresa; en este sentido, la inteligencia en la empresa ayuda a un directivo a orientarse adecuadamente en la elaboración de estrategias o tácticas para contrarrestar las fortalezas de la competencia y aprovecharse a la vez de sus debilidades.

Como se mencionó en la parte introductoria del presente artículo, la inteligencia en sus orígenes ha estado muy ligada al campo de la defensa o al campo militar, en la actualidad los estrategas, por la alta competencia que se presenta en el mercado, han tomado posiciones favorables con respecto a esta disciplina, considerando de urgencia extrema su aplicabilidad para enrumbar los negocios a mejores escenarios de competitividad. En el libro de Gordon Sullivan y Michael Harper ${ }^{(1)}$, dos expertos generales del ejército norteamericano, se aprecia claramente como la doctrina que se aplica para salir victorioso en conflictos militares es llevada a la guerra por ganar la supremacía en el complicadísimo mundo comercial.

En la carátula de los autores señalados que lleva por título La esperanza no es un método se lee lo siguiente: "Los ejecutivos norteamericanos aprendieron buena parte de sus técnicas generales de las prácticas de los militares estadounidenses durante la Segunda Guerra Mundial, ¡es notable que el ejército de los Estados Unidos que reinventó tanto su sistema de gerencia como su cultura de liderazgo esté otra vez guiando a la industria en la creación de nuevos enfoques en los retos gerenciales;".

En cualquier conflicto armado, ya sea de mediana o gran intensidad, si la inteli- 
gencia no está presente en el diseño de estrategias o tácticas, o en todo caso carece de oportunidad, el éxito de las operaciones será una remota posibilidad, mejor dicho lo más próximo será el triunfo del enemigo o del adversario.

Sun Tzu ${ }^{(2)}$ en el libro el Arte de la Guerra hace clara mención sobre este aspecto. $Y$ dice lo siguiente: "Conoce a tu enemigo y conócete a ti mismo; en cien batallas, nunca saldrás derrotado. Si eres ignorante de tu enemigo pero te conoces a ti mismo, tus oportunidades de ganar o perder son las mismas. $\mathrm{Si}$ eres ignorante de tu enemigo y de ti mismo, puedes estar seguro de ser derrotado en cada batalla".

Sin duda, la inteligencia aplicada a los negocios, es una de las columnas del mercado actual, al igual que la inteligencia llegó al campo militar para quedarse por miles de años, así también la Inteligencia Comercial o de los negocios llegó para quedarse; ésta siempre ha existido, de hecho, de alguna forma la ley de oferta y demanda es Inteligencia ya que cuando existe la necesidad del producto, puede ser entendida como una debilidad del comprador, aprovechándose esta debilidad con el incremento del precio; obviamente esta representación puede ser más compleja, pero este esquema sencillo demuestra que los negocios o comercios son tan antiguos como la guerra.

Si cambiamos nuestra visión de una guerra por el de negocios sin perder el sentido mismo de éste primero, habremos obtenido una ventaja considerable sobre nuestros competidores, para empezar, ¿Quién sería el «enemigon?... nuestra competencia, ¿dónde se libra la batalla?... en el mercado, ¿¿cuál es el botín de guerra?... los consumidores. Continuando de esta manera se llega a la necesidad de una estrategia.
Para atacar el mercado de una ciudad, sería estratégico saber cómo es el conjunto de consumidores, cómo están estadísticamente dispersas sus riquezas y sus necesidades de consumo, cómo están distribuidos geográficamente estos consumidores y cuál sena nuestra estrategia para llegar a ellos rápido y a bajo costo. Este tipo de preguntas puede ser respondido enviando un ejército de vendedores y encuestadores o puede ser respondido enviando un operativo de inteligencia para obtener información, procesarla y analizarla, de eso se trata esto. La primera estrategia pertenece al ejército, la segunda a la inteligencia.

Otro aspecto interesante es darnos cuenta de que una vez que obtuvimos información, ésta debe ser puesta en nuestro contexto, es decir, debemos relacionarla con nuestra propia información, y para ello debemos procesarla y analizarla, donde nuestra información y la obtenida deben estar en el mismo formato estándar para poderlas comparar y obtener nueva información verídica, para volver a planear nuevas estrategias o evaluar las existentes, atacar y apoderarnos eficientemente del mercado, esto es: Inteligencia Comercial, Económica o de Negocios.

¿Pero qué se entiende por inteligencia militar?, ¿cómo se entrelaza con la inteligencia comercial o de negocios?

Inteligencia Militar: son los conocimientos adquiridos a través de los esfuerzos de búsqueda, evaluación e interpretación de toda la información disponible que tiene que ver con un enemigo real o hipotético o con ciertas áreas de operaciones, inclusive las condiciones metereológicas y el terreno. Comprende también las deducciones hechas sobre las capacidades actuales y futuras del enemigo; las vulnerabilidades y los posibles cursos de acción que tenga a su alcance y que pueden afectar el cumplimiento de nuestra misión. 
En este concepto hay palabras claves, como: información disponible, capacidades, vulnerabilidades posibles cursos de acción, entre otros, que, día a día, son utilizados por el nivel estratégico de las empresas para montar eficaces estrategias de ataque o de defensa en este mundo globalizado.

Ahora, ¿cómo se puede diseñar un concepto amplio de inteligencia en los negocios, tomando como punto de partida la inteligencia militar o el que es utilizado por los organismos de defensa? Aquí el concepto:

Inteligencia de los negocios: son informaciones adquiridas que debidamente analizadas, procesadas, permite al nivel estratégico de un negocio tomar conocimiento de las posibles formas de acción que podría tener la empresa considerada como competencia, situación que le permitiría diseñar e implementar estrategias dirigidas a neutralizar dichas acciones, con lo cual el negocio mantendría intacto su poder de competitividad en el mercado.

Inteligencia competitiva: también está estrechamente ligada a la operatividad de la empresa, considerándose mayormente como sinónimo de inteligencia empresarial. Aquí dos conceptos:

- Inteligencia competitiva como “... el monitoreo coordinado y dirigido de los competidores ..., con la determinación de lo que harán, antes de lo que hagan,... ga- nar conocimiento anticipado de sus planes para planear las propias estrategias en consonancia".

- Inteligencia competitiva como “... la finalidad básica de determinar implicaciones para las operaciones estratégicas de la empresa a través de la identificación de las oportunidades y las amenazas del entorno competirivo". Porter identifica el "entorno competitivo", con lo que él llama "las cinco fuerzas competitivas" a saber: nuevos ingresos (competidores potenciales), las amenazas de los productos o servicios sustitutos, el poder negociador de los proveedores, el poder negociador de los clientes y la rivalidad de los competidores reales; de estas fuerzas, cuatro tienen expresión directa en las organizaciones y una (los productos o servicios sustitutos, pueden ser conocidos) si se conocen las organizaciones que lo producen y/o distribuyen.

Otros tratadistas lo conocen como inteligencia económica y lo definen de la siguiente manera: "Conjunto de las acciones de búsqueda, de tratamiento, de difusión y de protección de las informaciones útiles a los diferentes actores económicos. Estos actores son concebidos como un sistema global dedicado a inspirar la estrategia de la gerencia general de la empresa, tal como para informar en forma continua e inervar sus diferentes niveles de ejecución a fin de crear una gestión ofen-

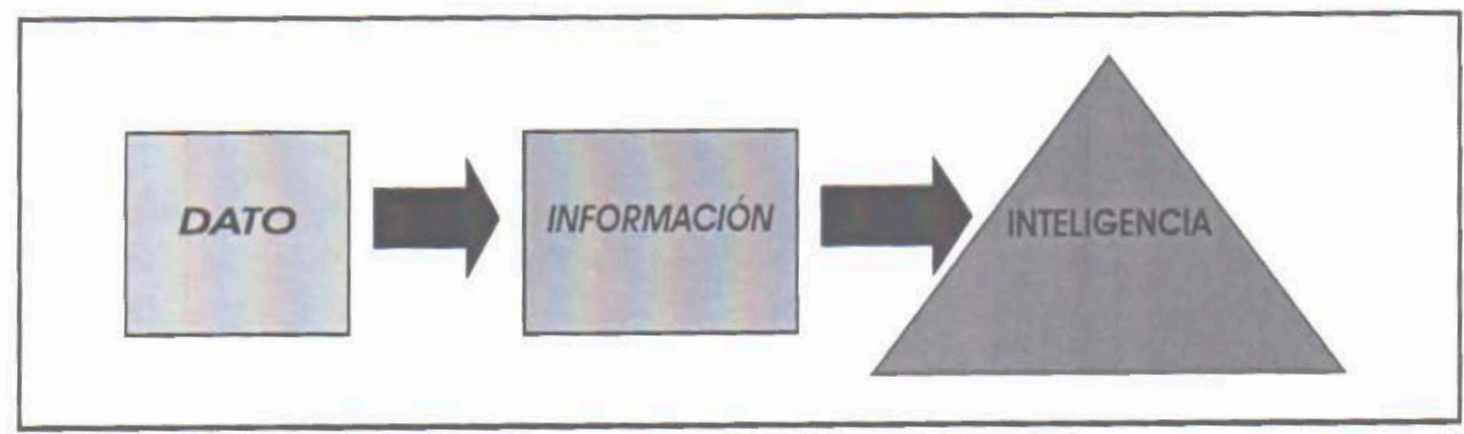


siva y colectiva de la información, la cual se convierte en su principal riqueza.

Como se puede apreciar, el concepto de inteligencia de negocios, competitiva y económica está referido al tratamiento de la información para ser utilizado con fines estratégicos en las organizaciones.

Para captar información, es sumamente importante la orientación del Esfuerzo de Búsqueda (EE.OO.BB), que debe estar bien dirigida. La empresa que aplica inteligencia sólo debe esforzarse en captar información valiosa para sus asuntos estratégicos. Si trata de elevar su poder de competencia, sería importante que el OO.EE.BB esté dirigido a obtener información sobre las fortalezas y debilidades del competidor, con el propósito de que el primero, con estrategias competitivas, sean debidamente vulneradas y la segundas sean aprovechadas en toda su amplitud. Así como en el campo de batalla, antes de ejecutar una acción bélica es importante conocer al enemigo en todos sus componentes, al menos eso es lo que dice la doctrina militar; en el mundo empresarial, los negocios también están obligados a tomar la misma posición sin dar tregua al adversario (competidor), que como es lógico, también éstos, están a la expectativa del menor error de quien consideran un eminente peligro para sus intereses económicos.

\section{PRINCIPIOS BÁSICOS DE LA INTELIGENCIA EN LA EMPRESA}

- UTIL: La inteligencia de tipo táctica, como estrategia, debe responder a los objetivos estratégicos de la empresa y con ello tiene que coadyuvar a que ésta logre su misión y visión. Debe permitir que los negocios obtengan mayor capacidad competitiva en el mercado.

- OPORTUNA: La inteligencia debe estar lista en el momento que lo necesite el elemento decisor. Un producto derivado del trabajo de inteligencia pierde vigencia, cuando se ha perdido la oportunidad de aplicarla. Por ejemplo, "Un producto derivado del ciclo de inteligencia puede ser inútil aplicarlo mañana para contrarrestar una amenaza, cuando se le necesitaba para ayer para el mismo propósito"

- FLEXIBLE: La inteligencia debe tener carácter predictivo, pero debe dejar un espacio para que el analista pueda corregir y darle continuidad, introduciéndole por cierto al ciclo de inteligencia. Como todo pronóstico, siempre existe una probabilidad de desacierto, pero ésta debe ser mínima cuando más agudo y preciso sea el trabajo de inteligencia. Ejemplo, “ ... la empresa SATURNO, que representa la competencia más fuerte para nuestra em-

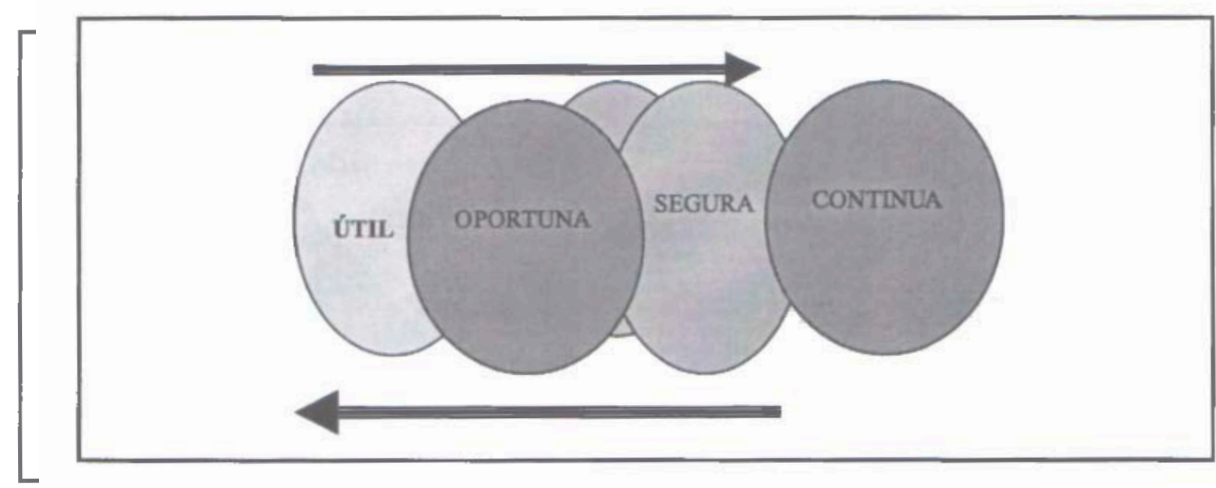

Figura 2. Principios básicos de Inteligencia que se deben practicar en todo negocio. 
presa, cuenta con capacidad financiera y tecnológica para realizar una alianza estratégica con la empresa Beta localizada en ALASKA; situación que podría afectar los intereses de nuestra compañía, que está interesada en seguir expandiéndose en este mercado; hecho que además podría vulnerar nuestra capacidad competitiva”. Esta hipótesis es una apreciación de inteligencia de tipo predictiva.

El analista utilizando, información disponible, que podría ser de fuente cerrada como abierta, está pronosticando lo que podría sucederle a su empresa, si Saturno y Beta se unen para operar en Alaska. Está pronosticando que su empresa perdería capacidad competitiva, que como es obvio le afectaría a toda su estructura empresarial. Para que no suceda lo que el analista está pronosticando, los niveles estratégicos tienen que tomar las medidas del caso para minimizar, en el mejor de los casos, o para neutralizar los impactos de esta inminente alianza comercial.

- SEGURA: la inteligencia tiene que tener un elevado carácter predictivo; la metodología utilizada para buscar la verdad debe conducir que el resultado de la inteligencia utilizada tenga la mínima proba- bilidad de fracaso. Por ello la empresa debe de preocuparse de optimizar el trabajo en todo el Ciclo de Inteligencia, desde el esfuerzo de la búsqueda de información hasta el producto de inteligencia propiamente dicho.

- CONTINUAS: en una empresa nunca debe de romperse el Ciclo de Inteligencia; éste debe ser continuo, predictivo, confiable y oportuno, a fin de que la toma de decisiones lleve a la empresa a un escenario de elevada competitividad.

\section{CICLO DE INTELIGENCIA EN LOS NEGOCIOS}

El ciclo de inteligencia incluye las siguientes actividades:

a. Recopilación y estructuración de información: La captación de información para producir Inteligencia en la empresa debe cubrir las siguientes premisas:

- Satisfacer las necesidades de la alta dirección y otras áreas claves de la empresa.

- Aprovechar la información que se genera en otras actividades similares de complemento o colaterales.

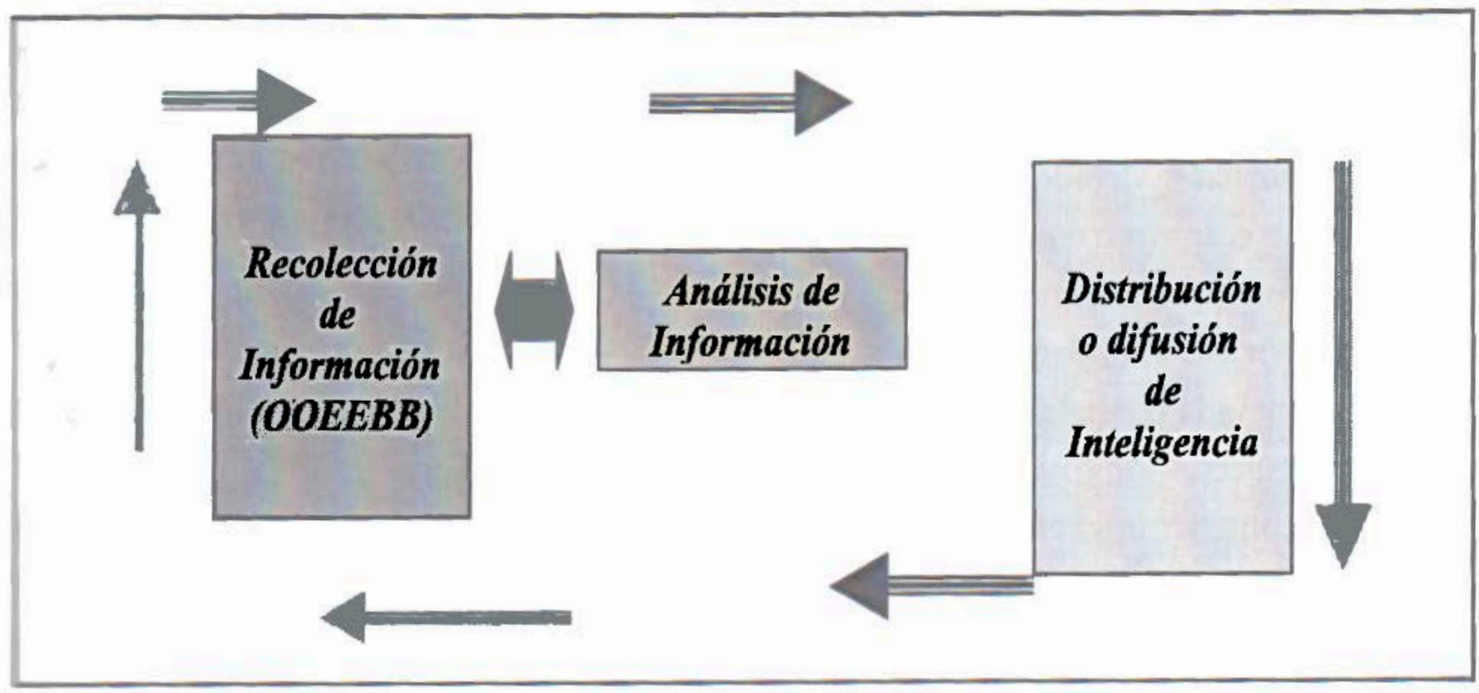


- Estructurar la información de manera tal, que sea de fácil acceso para su análisis.

Por ejemplo, en una empresa, la facilidad o dificultad que tenga para captar información dependerá de una serie de factores como son los tipos y tamaños de negocios, capacidad de los operadores de inteligencia para obtener información, preferentemente de fuente cerrada, (conduce a obtener información clasificada o de elevado valor), de las barreras a la información que coloque el adversario o competidor, del acervo tecnológico, entre otros. La implementación de todo un Sistema de Inteligencia requiere de una importante inversión, que está debidamente sustentada ya que la empresa elevará su potencialidad comperitiva.

\section{Los factores claves son:}

- Regionalidad: mientras más local sea el territorio de ventas y operaciones de una empresa, más fácil será encontrar información sobre ella.

- Dinamismo: mientras más dinámica y activamente crezca una empresa, en un mercado, éste le permitirá con mayor facilidad captar información, aunque podría disminuir el grado de precisión, por lo que se recomienda que el análisis de inteligencia debe ser más riguroso y científico.

- Especialización: mientras más especializada sea la empresa, mayor será el acceso a la información, debido a que sus relaciones con el entorno tenderán a ser más fluidas y confiables; elementos que le permitirán obtener abundante información, pero de elevada confiabilidad. Estos dos últimos aspectos son condiciones necesarias para obtener inteligencia de elevado valor para el negocio.
- Integración: las empresas altamente inregradas en sólidos procesos brindan poco margen al acceso a la información, es decir, se constituyen en un bloque sólido que impedirán o minimizarán el riesgo con respecto a la fuga de información. En este caso, el trabajo del adversario para obtener información será poco eficaz, ya que su trabajo de inteligencia será contrarrestado por distintas barreras que colocará la empresa para salvaguardar su información. Esto es lo que se conoce como acciones de contrainteligencia.

\section{b. Fuentes de información}

- Fuentes formales

- Fuentes alternativas

Una fuente formal es a la que ordinariamente se acude cuando se busca información relativamente facil de encontrar y por lo regular es posible adquirir o consultar. Y si la búsqueda no es tan simple, se tiene que buscar fuentes alternativas de información.

Las fuentes alternativas de información son las que el analista logra crear con su habilidad, creatividad y persistencia utilizando siempre las mismas fuentes formales ya conocidas.

Tanto las fuentes formales, como las alternativas, pueden ser primarias o secundarias; una fuente primaria es una fuente original de información, una fuente secundaria es la contenida en la bibliografía especializada. Cuando buscamos información normalmente debemos preguntarnos:

- ¿Dónde puede ser recogida la información?

- ¿Quién ordinariamente utiliza esta información?

- ¿Cuáles son las alternativas o dónde puedo localizarlas? 
Philip Kotler, en su texto cómo crear, ganar y dominar los mercados ${ }^{(3)}$, se fórmula la siguiente pregunta: QQué necesita saber una compañía acerca de sus competidores? Necesita conocer sus objetivos, estrategias, ventajas, desventajas y las conductas de respuesta. Además, formula algunas preguntas sobre la competencia para orientar su respuesta e insertarla en el ámbito de la inteligencia competitiva.

Preguntas, que todo analista de inteligencia empresarial debe hacer sobre el competidor para orientar su esfuerzo de búsqueda:

- Objetivos

¿El competidor está persiguiendo básicamente la rentabilidad actual?, ¿una mayor participación en el mercado?, ¿el liderazgo tecnológico?, ¿̨el competidor está interesado en hacer incursiones agresivas o está más interesado en la coexistencia?

- Estrategias

¿Cómo está tratando de ganar el competidor? ¿con los precios más bajos?, ¿¿la calidad más alta?, ¿el mejor servicio?, ¿los costos más bajos?, ¿las acciones del competidor están básicamente orientadas al corto o al largo plazo?

- Ventajas y desventajas

¿Cuáles son las principales ventajas del competidor en relación con nosotros?

¿Cuáles son las principales desventajas del competidor que nosotros podemos aprovechar?

- Conducta de respuesta

¿Cómo respondería el competidor si nosotros aumentáramos nuestros precios?, ¿y si bajáramos nuestros precios?, ¿ cómo respondería el competidor si aumentáramos agresivamente nuestro presupuesto de promoción o nuestra fuerza de ventas?
Philip Kotler argumenta que las compañías intentan reunir información para producir inteligencia de varias maneras:

Primero: examinan los periódicos, las revistas o cualquier otro material impreso en busca de información acerca de los competidores, estudian los anuncios publicitarios, el embalaje y los mensajes de los competidores. Generalmente contratan servicios de recortes periodísticos para este propósito.

Segundo: estudian las páginas de los competidores en Internet, que podrían incluir información detallada acerca del producto y los precios, información sobre nuestros productos, valores y políticas de la compañía, e información acerca de la ubicación de las empresas, oficinas, distribuidores y centros de servicio.

Tercero: contratan personal de un competidor en concreto para que ayude a la compañía a comprender la mentalidad de sus ex empleadores y probablemente sus iniciativas y reacciones.

Cuarto: encuestan a su personal de ventas y a sus intermediarios sobre sus impresiones y experiencias con un competidor concreto.

Quinto: mediante la referenciación (benchmarking) comparan el rendimiento propio con el del competidor, también adquieren y desarman el producto del competidor para ver como está hecho y analizar sus costos.

Los negocios han establecido una oficina de inteligencia competitiva que reúne información sobre el competidor y asesora sobre lo que se podría esperar en una batalla competitiva. Otras empresas, designan gerentes, expertos en competidores para asesorar a otros gerentes que necesitan información y tomar decisiones de nivel estratégico. 


\section{c. Análisis e interpretación de la infor- mación}

La inteligencia en la empresa además de ser un proceso deductivo (de lo general a lo particular) tiene sus raíces en un número de diferentes disciplinas complementarias: gestión de información, estadística, contabilidad, informática, matemáticas y armar rompecabezas.

La más rara de las disciplinas mencionadas, es armar rompecabezas, que significa simplemente habilidades para tomar lo que inicialmente parecen partes de la información sin relación que provienen del entorno y de las debilidades internas de las empresas competidoras que comparándolas encajan una sobre otra, originándose eslabones lógicos y coherentes, permitiendo dar una visión propia y completa de una situación determinada a los directores y ejecutivos, para posibilitarles una oportuna toma de decisiones. El analista de Inteligencia debe de sacudirse de todo tipo de prejuicios, de tal forma que el producto que obtenga, después de seguir todo el proceso de inteligencia, sea de lo más puro y lejos de cualquier contaminación, de no ser así, el analista podría inducir que los niveles estratégicos de los negocios tomen decisiones erradas, con alto costo para la empresa.

Para empezar un análisis, exprima información propia y ajena, y dótelos de oportunidades, compárelos, conviértalos en conocimiento y haga que éste tenga implicaciones para su empresa. Entonces estará haciendo inteligencia.

\section{d. Distribución o difusión de inteligencia}

Debe de quedar claro que lo que debe distribuirse es inteligencia y no informa- ción. Esta distribución debe ser a los furcionarios claves de la empresa.

La inteligencia obtenida, tal como se ha explicado en los principios básicos, debe de llegar en forma oportuna a los niveles estratégicos, pues, su distribución debe ser a través de canales ágiles, y altamente confiables; ya que no debe de olvidarse que se está distribuyendo información confidencial.

La inteligencia obtenida para su distribución tiene que dársele la categoría correspondiente para darle seguridad. La inteligencia puede ser de carácter reservado, secreto, confidencial, llaves que aseguraría que no sea utilizado por personas que no tienen nada que ver con esta parte del ciclo de inteligencia.

\section{LA FUNCIÓN DE INTELIGENCIA}

El desempeño de la función de inteligencia es y seguirá siendo estructuralmente la misma, pero las formas de llevarla adelante evolucionan y, como se ha expuesto, deberán adecuarse a las evoluciones rápidas del mercado, porque una simple actitud de reacción (sobre todo tardía) constituye un fracaso por anticipado, y no será posible revertirlo de modo alguno. Información es lo que hoy abunda, certeza y verdad es lo que cada vez escasea con acuciante desesperación. Los datos están cada vez más cerca de los usuarios, pero lo que se debe saber es la mejor forma de conjugación entre la voluntad de los protagonistas de los hechos y las voluntades que intervienen en el desarrollo de su discurrir.

Es un hecho que las máquinas aún no leen la mente, pero lo que se está experimentando en los países más desarrollados es la capacidad de simulación y correlación de escenarios, lo que permite enfrentar situaciones cada vez más impredecibles; en consecuencia, si los 
analistas y directores no entienden lo que se obtiene de la selección de entre millones de datos, se hará cada vez más difícil asistir a los decisores para explicarles las implicancias de un problema determinado, dada la complejidad de lo emergente, la creciente interconexión entre situaciones superpuestas y la sobreespecialización de los subcampos de análisis. La única alternativa es un mejoramiento sustantivo de la organización y funcionamiento de los constituyentes de la estructura de inteligencia, su dinámica y la sinergia de su desempeño ${ }^{(4)}$.

La función de inteligencia está soportando el mayor y más dramático desafío de la historia, desde que hace milenios, los antiguos maestros chinos sentaron las bases para el pensamiento analítico y dieron inicio a la carrera por entender la esencia de la verdad, para una eficaz toma de decisiones y triunfar con menores pérdidas en todo tipo de conflicto. Por ahora, el campo emergente de la guerra de informaciones y su soporte tecnológico es la economía, en todas sus escalas, en especial porque hay pocas limitaciones para prospectar en dicho campo, no se refiere al campo de la defensa convencional y toca un aspecto de la vida humana, tanto por sostener las finanzas que realimentan el desarrollo tecnológico, como por la importancia específica que conlleva el surgimiento de nuevos instrumentos en todos los campos de acción del poder, sean éstos de mayor o menor gravitación específica. Seguridad Nacional y Seguridad Internacional se sobreimponen y allí también la inteligencia debe decir más. El enfoque es ahora de naturaleza prospectiva, proactiva, horizontal, holística, pero sobre todo orientado hacia la búsqueda de las interconexiones entre la variedad creciente de variables intervinientes, que se agolpan en los escritorios de los analistas y en la mente de los decisores.
Por otro lado, cabe señalar que la revolución informática ha sido y sigue siendo un elemento casi deshumanizante, lleno de trampas y peligros que hasta la comunidad de inteligencia debe sortear, si desea seguir siendo vigente. Un argumento consecuente es la aceleración de los ciclos informativos debido a la influencia informática; así, la velocidad de la obsolescencia de los modelos y métodos y la velocidad de requerimiento se sobreimponen una y otra vez, por obra del avance tecnológico, haciendo más factible ayudar a los analistas de inteligencia. Se vive una vorágine de la cual sólo resta un peldaño por conocer, la completa inteligencia artificial, donde máquinas programen a otras máquinas y donde la brecha de accesibilidad entre países desarrollados y los otros, en vías de desarrollo, se aprecie dramáticamente inalcanzable. Eso también será motivo de nuevos conflictos de intereses y allí deberá estar como siempre la inteligencia, buscando lo que otros no encuentran y entendiendo lo que las mentes simples sólo registran; viendo lo que simples ojos y oídos sólo miran y escuchan, pero no oyen ni ven.

Para continuar cumpliendo con los roles esenciales de recolectar, procesar y difundir inteligencia-producto, los nuevos modelos e ideas sobre el trabajo analítico deben de considerar algunos aspectos dinámicos de la vida contemporánea:

* Una sucesiva mutación geoestratégica, entendida como un replanteamiento de ideas y contextos que, en ese ámbito, se aceptaban como inmutables y que en la realidad pueden llegar a ser un obstáculo para entender la nueva complejidad del mundo contemporáneo.

* Un progresivo replanteamiento de la utilidad de la prospectiva, no sólo en el plano metodológico, sino en los enfoques 
culturales, sociales y tecnológicos que debieran expandir su ámbito, para ganar en oportunidad y certeza.

* Una creciente vigencia del concepto Geocultura, que permite asociar con mayor precisión los ambientes planetarios y las expresiones creativas de los habitantes, para entender con mayor acierto el discurrir de situaciones que poseen sus propios relojes y calendarios para expresar la significación estratégica de su desenvolvimiento.

No obstante, en el avance vertiginoso de la tecnología, es el analista quien sigue ordenando y priorizando lo que las máquinas a lo sumo clasifican. En consecuencia, es indis- pensable establecer un nuevo planteamiento de ideas, conceptos operacionales y organizacionales para el diseño de nuevas formas de trabajo en la fase general de procesamiento de datos e informaciones. Liderazgo y conducción, respaldados por una concepción estratégica, en equilibrio con estructuras, procesos y sistemas operacionales, alumbrados por los principios de la organización de pertenencia, serán elementos y factores que cobrarán su efectivo valor en la medida que la calidad del personal de analistas demuestre condiciones de excelencia, experiencia y preparación, apoyadas en una vocación profesional incuestionable (ver figura 3).

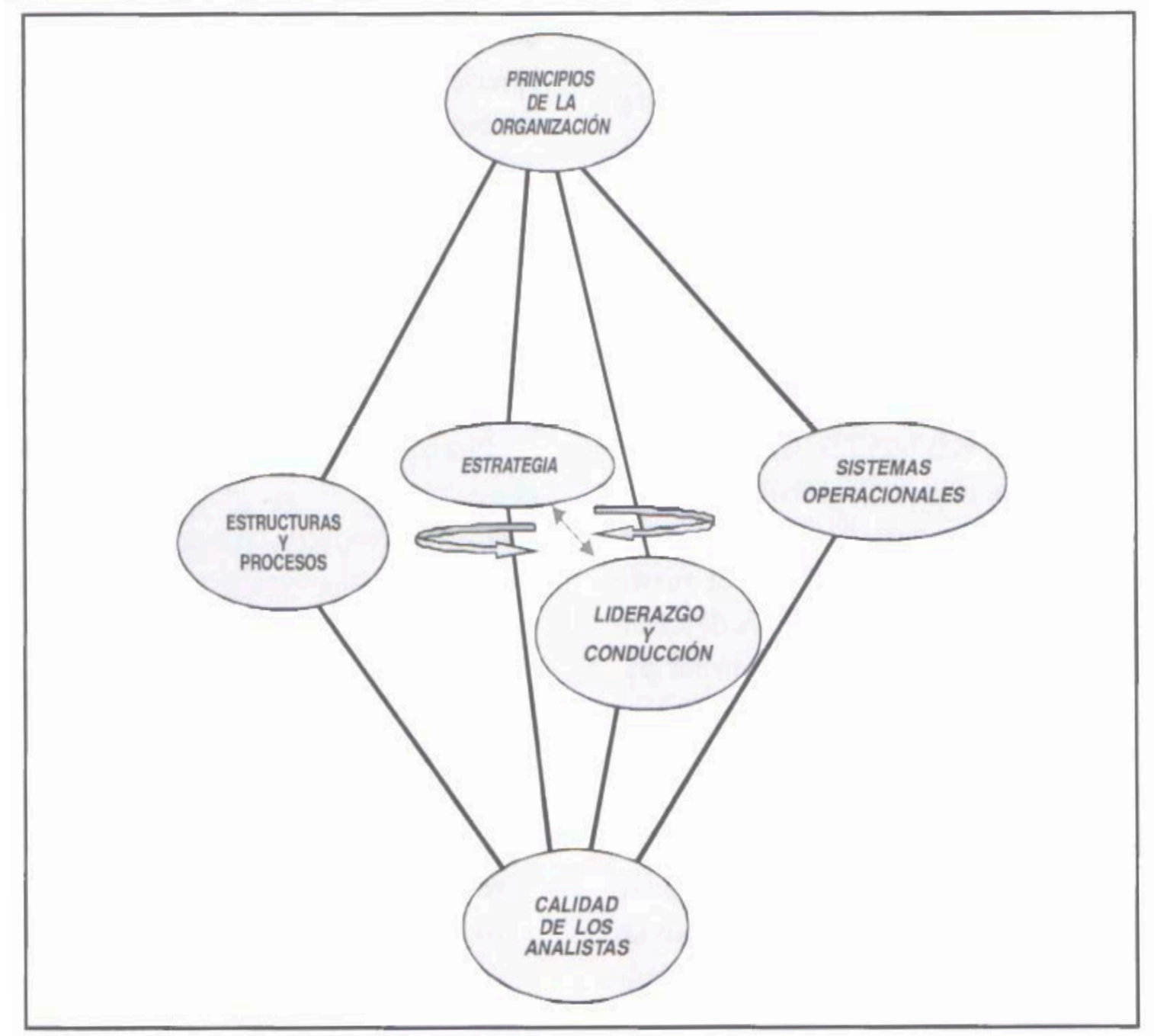


Ésa parece ser la única conclusión preliminar, en una línea de estudio del fenómeno analítico de inteligencia que no deja se ser estimulante gracias a los permanentes desafíos del entorno, situaciones de inestabilidad permanente, que la vorágine del discurrir del gran juego estratégico mundial plantea de manera permanente.

\section{INTELIGENCIA ECONÓMICA. ALGUNAS EXPERIENCIAS A NIVEL DE GOBIERNOS}

Los Organismos de Inteligencia (OI) han recolectado y analizado información económica por casi cincuenta años. Esta actividad centra su atención en aquellas áreas que podrían afectar los intereses nacionales, incluyendo las economías de países extranjeros, las tendencias económicas mundiales, e información para el apoyo de negociaciones comerciales.

Aproximadamente el $95 \%$ de los análisis sobre tópicos económicos deriva de fuentes abiertas; no obstante, el otro $5 \%$ suele ser inaccesible y debe ser cubierto por los $\mathrm{OI}$ a través de actividades de contrainteligencia.

En EE.UU., el rol de la inteligencia en el componente económico se está orientando a la obtención de información referida a prácticas comerciales desleales que perjudiquen a firmas norteamericanas -colocándolas en una posición de desventaja para la obtención de algún contrato con un gobierno extranjero-, $o$ a situaciones en las que algún gobierno extranjero esté involucrado, de algún modo, en dicha transacción desleal.

Esta información es remitida a los departamentos de Estado y Comercio que son los responsables de determinar la utilización o no de la información de inteligencia y, en el caso afirmativo, el momento apropiado considerando, entre otras cosas, sus efectos en las relaciones bilaterales involucradas. Asimismo, son los responsables de establecer las acciones diplomáticas correspondientes con el gobierno implicado en tal situación.

Otra de las cuestiones relacionadas con la inteligencia económica que despierta puntos de vista encontrados, es la referida a si los Organismos de Inteligencia deben proveer apoyo de inteligencia estratégica y táctica directa al sector privado. Según Roy Godson ${ }^{(5)}$, esta idea se ve justificada, algunas veces, por un intento de definir los diversos desafíos económicos como nuevas amenazas a la Seguridad Nacional, dignos de ser tratados con los instrumentos tradicionales empleados en mantenerla; tales como el apoyo de Inteligencia.

A criterio del citado autor, la temática debería ser considerada como desafíos económicos y no como amenazas. Si bien reconoce la importancia del tema económico, sostiene que un programa de apoyo directo al sector privado implicaría una serie importante de problemas prácticos, desde cómo realizar su implementación hasta las consecuencias de hacerlo de un determinado modo; seleccionar qué sector o compañía será asistida, quién lo determina y sobre qué bases.

Respecto a los métodos, la inteligencia ha reunido información económica para investigar las tendencias globales y regionales, con lo que podría considerarse la utilización de procedimientos legales. No obstante ello, actualmente el conflicto se incrementa por la utilización de información obtenida por medio del espionaje industrial en prácticas comerciales inequitativas. Muchos Estados utilizan el espionaje económico para captar información y producir inteligencia sobre industrias específicas y la comparten con productores nacionales, para tratar de suplir las ventajas competitivas de otras compañías en el mercado global. 
Conforme a numerosos artículos de prensa y a lo descrito por Peter Schweizer en su libro Espionaje Amigable, Francia ha utilizado los recursos de sus Servicios de Inteligencia para obtener información económica secreta de otros países, así como también lo han hecho Israel, Alemania, Japón y Corea del Sur. De acuerdo a lo afirmado en dicha publicación «Un análisis realizado sobre 173 naciones determinó que 57 de ellas habían tratado de conseguir secretamente tecnologías de avanzada provenientes de corporaciones de EE.UU.».

El proceso de reformulación de los esfuerzos y recursos de la inteligencia originó un debate referido a definir o no el apoyo a la competitividad económica como un nuevo requerimiento de Inteligencia.

En este debate, merece tenerse en cuenta la opinión de Randall Fort, quien afirma que: «Existe mucha confusión con respecto a la inteligencia económica, y gran parte de la misma se deriva de una confusión terminológica. Espionaje económico, espionaje industrial, espionaje comercial y otras expresiones similares pueden hacer o no referencia a la misma cosa, pero son utilizadas frecuentemente de manera indistinta».

Asimismo, señala que existen errores de interpretación con respecto a los roles apropiados que se le asigna a la inteligencia económica, comparados con aquellos que desarrollan otras funciones de inteligencia.

A su entender existe un rol histórico y legítimo de la Inteligencia económica en el apoyo a los decisores. Aunque la política económica ha adquirido más protagonismo recientemente, ha sido un área importante en la recolección y el análisis de Inteligencia durante muchos años.

Fort detalla tres áreas en las cuales los OI han estado tradicionalmente activos en lo que respecta al componente económico:

* El aporte de información elaborada como herramienta de apoyo a los gobiernos para establecer la política económica. Este respaldo ha incluido el análisis de negociaciones bilaterales y multilaterales, la identificación de tendencias económicas, el análisis sobre los propósitos de competidores económicos, la integración de enormes volúmenes de datos en una imagen completa de los factores políticos y económicos que afectan la estabilidad internacional, y facilitar a los decisores el entendimiento de las reglas del juego económico cuando es desarrollado por otros (monitorear subsidios externos, lobbys, alianzas, restricciones importantes, entre otras).

* El seguimiento de las tendencias internacionales sobre avances en tecnología que pudieran afectar la Seguridad Nacional. El Gobierno necesita estar informado sobre los desarrollos en computadoras, semiconductores, telecomunicaciones, y todo avance tecnológico que pudiera afectar, entre otras cosas, las capacidades militares, económicas o cualquiera de los intereses de Seguridad Nacional.

* La contrainteligencia económica, cuya función consiste en la identificación y neutralización del accionar de Servicios de Inteligencia extranjeros cuyo objeto de interés esté constituido por individuos o compañías determinadas, con el fin de robar información y/o tecnología para ser utilizadas en beneficio propio. 


\section{CONCLUSIONES}

1. Inteligencia, en cuanto a su aplicación, ha traspasado las fronteras del ámbito militar o del campo de la Defensa Nacional. En la actualidad esta disciplina es utilizada por organizaciones empresariales como medio para lograr, no sólo contrarrestar las estrategias de su competidor o adversario, sino también para poder posicionarse de mercados, donde el competidor en una primera instancia, tenía la supremacía absoluta.

2. El mundo de los negocios de hoy exige una aplicación cada vez más eficiente de la información disponible. La inteligencia de negocios genera un conocimiento a las empresas, que se deriva de la correcta utilización de la información generada dentro y fuera de la empresa. Es una herramienta que pone a disposición de los usuarios la información correcta en el lugar correcto. Son múltiples los beneficios que ofrece a las empresas, entre ellos se encuentra la generación de una ventaja competitiva.

3. Inteligencia económica, comercial o competitiva, exige seguir todo el proceso del Ciclo de Inteligencia, para que los productos de este proceso sean de la más alta confiabilidad y así la toma de decisiones renga un elevado grado de precisión o eficacia. El Ciclo de Inteligencia en las organizaciones tiene que ser continua, para dar cumplimiento al principio de oportunidad.

4. Si las empresas quisieran mantener su liderazgo en el mercado se recomienda que implementen todo un sistema de inteligencia económica, sólo así estarán en capacidad de contrarrestar o neutralizar cualquier amenaza que pueda provenir del entorno.
5. Para el proceso de inteligencia en los negocios es importante tener información de fuente cerrada o la llamada clasificada. Con este tipo de información la empresa estará en capacidad de diseñar estrategias dirigidas no sólo a vulnerar las fortalezas del competidor o aprovechar sus debilidades, sino a lograr propósitos que le conlleven a obtener ventajas competitivas sólidas y sostenidas.

6. Los gobiernos también utilizan la inteligencia económica para coadyuvar al potenciamiento del sector empresarial. Es por ello que utilizan lo que se denomina el espionaje industrial como forma de obtener información estratégica y así utilizarla en sus planes de Gobierno, para ello montan todo un sistema de inteligencia apoyado por la tecnología informática como herramienta de búsqueda de información.

\section{REFERENCIAS}

1. Balbi, Eduardo Raúl. La inteligencia en apoyo de los procesos decisionales (algunas reflexiones preliminares), en: Revista de la Escuela Nacional de Inteligencia, República Argentina.

2. Gordon Sullivan y Michael Harper. La esperanza no es un método. Editorial Norma. Barcelona. 2000.

3. Verda, Horacio de. Producción de Inteligencia Estratégica. El método y las técnicas, en: Revista de la Escuela Nacional de Inteligencia, República Argentina.

4. R.H. Mathams. Análisis de Inteligencia: Guía del analista de inteligencia, traducción existente en la biblioteca de la Escuela Nacional de Inteligencia del libro Strategic Intelligence: Theory an Aplication, editado por Dearth-Goodden, segunda edición, 1995. 
5. Ugarte, José Manuel. Sistema Nacional de Inteligencia Argentino.

6. Kotler, Philip. El marketing, según Kotler, Editorial Paidós, Barcelona, 1999.
7. Kotler Philip. Marketing se mueve, una nueva aproximación a los beneficios, el crecimiento y la renovación. Editorial Paidós, España, 2002.

(1) Gordon Sullivan y Michael Harper. La esperanza no es un método, Editorial Norma, Barcelona, 2000.

(2) Sun Tzu fue un general chino que vivió alrededor del siglo V antes de Cristo. La colección de ensayos sobre el arte de la guerra atribuida a Sun Tzu es el tratado más antiguo que se conoce sobre dicho tema. A pesar de su antiguedad, esta obra domina sobre cualquier otra sobre el tema

(3) Philip Kotler. "El Marketing según Kotler". Cómo crear, ganar y dominar mercados. Edit. Paidós, Barcelona, 1979.

(4) Prof. Ulises A. Faúndez (M.A.), académico del Instituto de Ciencia Política Universidad de Chile.

(5) Roy Godson, experto en espionaje e inteligencia de la Universidad de Georgetown, en Washington, D.C. 